\title{
BMJ Open Use of smartphones for detecting diabetic retinopathy: a protocol for a scoping review of diagnostic test accuracy studies
}

\author{
Choon Han Tan (D) , 'Willie-Henri Quah, ${ }^{2}$ Colin S H Tan, ${ }^{3}$ Helen Smith, ${ }^{4}$ \\ Lorainne Tudor Car $^{1}$
}

To cite: Tan $\mathrm{CH}$, Quah W$\mathrm{H}$, Tan CSH, et al. Use of smartphones for detecting diabetic retinopathy: a protocol for a scoping review of diagnostic test accuracy studies. BMJ Open 2019;9:e028811. doi:10.1136/ bmjopen-2018-028811

- Prepublication history and additional material for this paper are available online. To view these files, please visit the journal online (http://dx.doi org/10.1136/bmjopen-2018028811).

Received 04 January 2019 Revised 05 November 2019 Accepted 14 November 2019

Check for updates

(C) Author(s) (or their employer(s)) 2019. Re-use permitted under CC BY-NC. No commercial re-use. See rights and permissions. Published by BMJ.

${ }^{1}$ Lee Kong Chian School of Medicine, Nanyang Technological University Singapore, Singapore, Singapore ${ }^{2}$ Brighton and Sussex Medical School, Brighton, UK ${ }^{3}$ Department of Ophthalmology, National Healthcare Group Eye Institute, Tan Tock Seng Hospital, Singapore, Singapore

${ }^{4}$ Family Medicine and Primary Care, Lee Kong Chian School of Medicine, Nanyang Technological University Singapore, Singapore, Singapore

Correspondence to

Dr Lorainne Tudor Car;

lorainne.tudor.car@ntu.edu.sg

\section{ABSTRACT}

Introduction Diabetic retinopathy (DR) is a common microvascular complication of diabetes mellitus and the leading cause of impaired vision in adults worldwide. Early detection and treatment for DR could improve patient outcomes. Traditional methods of detecting DR include the gold standard Early Treatment Diabetic Retinopathy Study seven standard fields fundus photography, ophthalmoscopy and slit-lamp biomicroscopy. These modalities can be expensive, difficult to access and require involvement of specialised healthcare professionals. With the development of mobile phone technology, there is a growing interest in their use for DR identification as this approach is potentially more affordable, accessible and easier to use. Smartphones can be employed in a variety of ways for ophthalmoscopy including the use of smartphone camera, various attachments and artificial intelligence for obtaining and grading of retinal images. The aim of this scoping review is to determine the diagnostic test accuracy of various smartphone ophthalmoscopy approaches for detecting DR in diabetic patients.

Methods and analysis We will perform an electronic search of MEDLINE, Embase and Cochrane Library for literature published from 2000 onwards. Two reviewers will independently analyse studies for eligibility and assess study quality using the QUADAS-2 tool. Data for a $2 \times 2$ contingency table will be extracted. If possible, we will pool sensitivity and specificity data using the randomeffects model and construct a summary receiver operating characteristic curve. In case of high heterogeneity, we will present the findings narratively. Subgroup analysis and sensitivity analysis will be performed where appropriate. Ethics and dissemination This scoping review aims to provide an overview of smartphone ophthalmoscopy in DR identification. It will present findings on the accuracy of smartphone ophthalmoscopy in detecting DR, identify gaps in the literature and provide recommendations for future research. This review does not require ethical approval as we will not collect primary data.

\section{INTRODUCTION}

Diabetic retinopathy (DR) is the primary cause of impaired vision in patients 20-74 years of age. ${ }^{1}$ It is a common microvascular complication of diabetes mellitus (DM): one-third of patients
Strengths and limitations of this study

- This is a protocol for a scoping review that aims to provide evidence to inform the use of smartphone ophthalmoscopy in patients with diabetes mellitus.

- Our findings may be of particular relevance to clinicians in rural or resource-constrained settings with limited access to standard diagnostic approaches for diabetic retinopathy (DR) identification.

- We will employ a scoping review approach to perform a comprehensive search of the literature and thorough data analysis, with the aim of providing reliable findings on the accuracy of smartphone ophthalmoscopy for DR identification.

- While not a requirement of scoping reviews, our review will include a quality assessment of the included studies; a potential limitation of this scoping review is the possibility of missing relevant articles.

with DM have signs of DR and of these people, one-third possess sight-threatening DR. ${ }^{2} \mathrm{DR}$ is classified into proliferative DR (PDR) and nonproliferative DR (NPDR). PDR is characterised by neovascularisation, that is, angiogenesis in response to retinal tissue hypoxia. Neovascularisation potentially results in preretinal and vitreous haemorrhage, leading to visual loss and tractional retinal detachment. It can also cause iris neovascularisation with resultant increase in intraocular pressure, which may eventually result in neovascular glaucoma. ${ }^{3}$ The typical clinical features of NPDR include: (1) microaneurysms due to weak capillary walls; (2) hard exudates from vascular protein leakage; (3) cotton wool spots, caused by ischaemic infarcts leading to fluid accumulation within the retinal nerve-fibre layer; and (4) haemorrhages from the rupture of weak capillary walls. Diabetic macular oedema (DME), the thickening of and fluid accumulation in the retina, can exist in both PDR and NPDR and may cause vision loss. ${ }^{4}$ 


\section{Rationale}

There are several effective treatments available for DR. They include vascular endothelial growth factor inhibitors, panretinal or focal photocoagulation, and vitrectomy. ${ }^{5-9}$ Strict glycaemic and blood pressure control can also prevent and slow the progression of DR. ${ }^{10}$ As these treatments are more effective at halting or delaying visual loss than reversing visual impairment, timely DR screening and early detection is imperative. Furthermore, as most DR patients have no symptoms until the disease progresses to advanced stages or DME occurs, detecting early DR, before irreversible changes to visual acuity occurs in late disease, is important. ${ }^{11}$

The gold standard for diagnosing DR is the Early Treatment Diabetic Retinopathy Study (ETDRS) seven-field stereoscopic colour fundus photography or fluorescein angiography performed by a qualified photographer and reader. ${ }^{12}$ However, fundus cameras are cumbersome, expensive, operator-dependent and limited to a doctor's office, often requiring patients to sit upright. ${ }^{13}$ This makes retinal imaging a challenging process for ill, wheelchair-bound or disabled patients. ${ }^{14}$ Moreover, fluorescein angiography is invasive and associated with significant side-effects. ${ }^{15}$ Thus, both gold standards are not pragmatic for screening in a regular clinical setting. Other accurate, ${ }^{16}$ acceptable and commonly employed approaches to DR screening and identification include: (1) ophthalmoscopy under mydriasis by trained healthcare professionals, for example, general practitioners (GPs), opticians, ophthalmologists, doctors specialising in diabetes care; (2) slit-lamp biomicroscopy; or (3) other forms of fundus photography. ${ }^{17}$ Optical coherence tomography is an emerging technology that can accurately identify macular oedema and measure retinal thickness ${ }^{18}$ but does not reliably grade the severity of DR.

Lately, with the immense advancement of mobile phone technology, there is a growing interest in the use of smartphones for DR identification. Smartphone ophthalmoscopy, the use of a smartphone's in-built camera for retinal imaging, could be a valuable approach to detecting DR due to its portability and ease-of-use. It can potentially optimise the frequency of screening, and increase compliance of DR patients in follow-up care. Furthermore, the operation of smartphone ophthalmoscopy in detecting DR may be open to all healthcare workers and not only to highly skilled healthcare professionals, further increasing likelihood of early detection. The captured images are subsequently transferred to an ophthalmologist or trained clinician for analysis. Additionally, smartphone ophthalmoscopy may be more affordable than a traditional fundus camera and is not restricted to the doctor's office. ${ }^{19}$ This is particularly relevant for rural areas or developing countries where fundus cameras and healthcare professionals may not be readily available. ${ }^{20}$ For example, all doctors and $97 \%$ of nurses surveyed in Nigeria's secondary healthcare facilities owned a smartphone, and most were aware of the concept of mobile health. ${ }^{21}$ Images obtained can also be graded remotely by trained graders using telemedicine platforms, further enhancing the advantages of screening using smartphone ophthalmoscopy.

Smartphone ophthalmoscopy may also allow for automated DR detection using smartphone-based automated analysis software based on artificial intelligence (AI). AI is the simulation of human intelligence by a computer software based on teaching the computer to recognise specific patterns. It is increasingly employed in a variety of tasks including accurate classification of high-resolution images. $^{22}$ There are a number of existing AI algorithms for analysis of retinal images from high-quality conventional fundus cameras. ${ }^{23} 24$ However, AI could also be coupled with the use of smartphone ophthalmoscopy. ${ }^{25}$ AI-based DR grading software may enable grading by all health workers and allow for higher efficiency, reproducibility and earlier detection of DR. Given its potential in reducing the burden of DR on healthcare systems, it is important to determine the sensitivity and specificity of smartphone ophthalmoscopy, with or without the use of AI, for detection of DR.

To our knowledge, there are no existing reviews evaluating exclusively the sensitivity and specificity of smartphone ophthalmoscopy for DR. A meta-analysis by Shi et al from $2014^{26}$ and a review by Horton et alfrom $2016,{ }^{27}$ both on the use of telemedicine for detecting DR, included studies evaluating diverse retinal imaging techniques and the transfer of digital images to a 'reading centre' for 'remote diagnosis'. In contrast, our scoping review aims to only include studies on digital retinal imaging using smartphones, with or without telemedicine. Additionally, literature reviews by Bolster $e t a t^{28}$ and Micheletti et $a t^{29}$ explored and discussed smartphone retinal imaging technology, without aiming to collate data or perform any analysis on its diagnostic test accuracy, and underscored the 'immense potential' of smartphone ophthalmoscopy. Furthermore, a review by Fenner et $a l^{30}$ examined the advances in retinal imaging in DR, including a section on the sensitivity and specificity of smartphone imaging modalities. However, our review aims to use a different, systematic, more transparent and focused methodological approach, such as in our search strategy. Lastly, a meta-analysis by Vilela $e t a l^{\beta 1}$ compared the agreement between smartphone ophthalmic images and retinal cameras. However, this systematic review included and pooled many eye diseases such as glaucoma, ocular hypertension and pseudophakia, or included patients from an emergency department or hospital setting without a prespecified condition. In contrast, our review focuses on diabetic patients.

\section{Objectives}

Our objective is to determine the diagnostic accuracy of smartphone ophthalmoscopy to detect the presence and degree of DR in patients with type 1 and $2 \mathrm{DM}$ by comparing their sensitivities, specificities, positive predictive value (PPV) and negative predictive value (NPV) with respect to a reference standard. Our secondary objectives include the investigation of 
potential sources of heterogeneity among studies and their impact on the diagnostic accuracy of smartphone ophthalmoscopy. As this is a novel research area with limited evidence, we aim to collate all relevant studies. We will therefore employ a scoping review approach ${ }^{32} 33$ and broad inclusion criteria.

\section{METHODS AND ANALYSIS}

This review will be conducted according to the Preferred Reporting Items for Systematic reviews and MetaAnalyses extension for Scoping Reviews checklist ${ }^{34}$ and the Cochrane Handbook for Systematic Reviews of Diagnostic Test Accuracy. ${ }^{35}$

\section{Inclusion criteria}

Smartphone ophthalmoscopy involves imaging the retina using a smartphone camera. This includes, but is not limited to, the following approaches:

1. Direct ophthalmoscopy: an adaptor is externally attached to a smartphone's camera. Adapters are devices containing lenses and equipment to modify the path and polarisation of light. They also contain an in-built light source to deliver coaxial retinal illumination. Adapters are designed to improve the picture quality of retinal imaging. ${ }^{28}$ Ideally, these adaptors are affordable, portable and simple to use. The specifications of each manufacturer differ: different products allow measuring of different field-of-view angles, and have different power and magnifications. A second method does not require the use of adaptors. Instead, it involves using an external light source mounted to the smartphone, to provide more optimal illumination of the fundus. Both photography and videography can be performed. ${ }^{36}$

2. Monocular indirect ophthalmoscopy: the autofocus function of the camera is used to capture retinal images, through a hand-held or externally mounted lens, with coaxial retinal illumination provided by the phone's light-emitting diode (LED). This method typically requires mydriasis. $^{28}$

We will include studies (both academic papers and grey literature) on participants with type 1 or $2 \mathrm{DM}$, and involving the use of smartphone ophthalmoscopy as pointof-care devices to detect the presence and/or degree of DR compared with a suitable reference standard or another form of index test. Such studies may employ the use of AI, look at a variable number of visual fields or use either the smartphone's intrinsic LED or an external light source. We will include studies in which smartphone ophthalmoscopy digital retinal images are read by health professionals as well as AI-based automated software.

As a reference standard, we will use the ETDRS seven standard fields stereoscopic colour fundus photography performed by a qualified photographer and reader. Studies using other commonly employed retinal imaging modalities as reference standards, such as direct or indirect ophthalmoscopy, slit-lamp biomicroscopy or other forms of fundus photography, will also be included. We will perform subgroup analyses based on the different reference standards used.

\section{Search method for the identification of studies}

Electronic searches will be done on the following databases without language restriction:

1. EMBASE (Ovid).

2. MEDLINE (Ovid).

3. Cochrane Library.

We will perform a librarian-assisted search for literature published from 2000 onwards as mobile phone features were limited prior to that. In our search, we will use a combination of medical subject headings and keywords relating to DR (eg, 'diabetic retinopathy', 'macular oedema' and 'diabetic maculopathy') and to smartphones (eg, 'mobile health', 'mobile phones' and 'applications') or AI (eg, 'artificial intelligence' and 'machine learning') (online supplementary data 1). We will also include eligible studies cited in known primary and review articles to identify additional studies missed by the electronic searches. For translation of articles written in other languages, we will rely on the international team at our institution.

\section{Study selection, data extraction and quality assessment}

The screening will be performed at two stages-title and abstract, followed by full text screening-presented in a Preferred Reporting Items for Systematic reviews and MetaAnalyses flow diagram. Both stages will be performed by two reviewers independently. Any disagreements will be resolved via discussion.

Data for assessing the diagnostic test accuracy of smartphone ophthalmoscopy will be extracted by comparing results between the reference standards and the smartphone ophthalmoscopic device, either directly or by backcalculating from sensitivity and specificity data provided. Thereafter, we will generate a $2 \times 2$ contingency table comprising:

1. True positives: both tests indicate the presence of DR.

2. True negatives: both tests indicate the absence of DR.

3. False positives: smartphone ophthalmoscopy indicates $\mathrm{DR}$, while reference standards indicate the absence of DR.

4. False negatives: smartphone ophthalmoscopy indicates the absence DR, while reference standards indicate DR.

Data will be entered into and analysed in the RevMan V.5.3 software from the Cochrane Collaboration, ${ }^{37}$ to provide a visual representation of sensitivity and specificity data through a forest plot. The nature and reasons for uninterpretable results will be recorded.

Additional data extraction to describe and consolidate the characteristics of each included study will be carried out using a prepiloted form (online supplementary data 2) by two researchers independently with disagreements resolved via discussion. We will extract the following data: 1. Study information: study author; date published.

2. Population characteristics: sample size; mean age and age range of patients; duration and type of DM. 
3. Information regarding smartphone ophthalmoscopy: imaging techniques; image resolution; smartphone used; adaptors used and their specifications, for example, field-of-view.

4. Healthcare professional performing smartphone ophthalmoscopy.

5. Reference standard used.

6. AI software used (if applicable).

The methodological quality of included studies will be assessed by the QUADAS-2 tool for bias and applicability, specifically concerning patient selection, index test, reference standard and flow/timing. ${ }^{38}$ QUADAS-2 is well-established and routinely employed in diagnostic test accuracy reviews. While study quality assessment is not mandatory in scoping reviews, several scoping reviews have performed quality assessments; such assessments may even be an important component of scoping reviews. ${ }^{39} 40$ Assessing the methodological quality is essential for: (1) determining if a study is sufficiently relevant for inclusion or produces valid and unbiased results; (2) influencing the strength of the evidence; and (3) performing sensitivity analyses. QUADAS-2 will be applied independently by two reviewers and any disagreements will be resolved through discussion.

\section{Data analysis}

From the tabulated $2 \times 2$ contingency tables, we will calculate, report and present the following outcomes-sensitivity, specificity, PPV, NPV and diagnostic OR, along with the corresponding 95\% CIs-of smartphone ophthalmoscopy in identifying DR. Heterogeneity would be evaluated using chi square and $\mathrm{I}^{2}$ (measure of inconsistency) tests. Due to the heterogeneity of data expected, the randomeffects model will be used for meta-analysis. Studies will be represented in forest plots. Sensitivities and specificities will be represented in a summary receiver operating characteristic curve, using RevMan V.5.3 software. In case pooling of studies is not possible due to high clinical or methodological heterogeneity, we will present the findings narratively.

If sufficient studies are included, publication bias will be analysed using Deeks' test. ${ }^{41}$ Subgroup analyses will be performed to identify potential sources of heterogeneity. We will explore the impact of the following covariates:

1. Different reference standards employed in the studies.

2. Different attachments or lenses used as part of smartphone ophthalmoscopy.

3. Different approaches to DR grading, that is, AI-based versus healthcare professionals.

4. Different severity of DR, for example, mild to severe NPDR, early to severe PDR, DME, referral-warranted or sight-threatening DR.

5. Patient population, for example, age, type and duration of DM.

6. Device operators, that is, differences across healthcare professionals.

7. Testing conditions, for example, mydriasis, room lighting.
Sensitivity analysis will be performed by comparing high-quality to low-quality studies, evaluating if significant differences in sensitivity and specificity exist between the two groups.

For all tests, statistical significance is defined as $p \leq 0.05$, except for Deek's test for publication bias $(p \leq 0.1)$.

\section{Patient and public involvement}

Patients will be neither recruited nor directly involved in the conduct of this scoping review. This review was informed by Vision 2020: The Right to Sight, a worldwide initiative by the WHO to eradicate preventable blindness. One strategy of Vision 2020 involves utilising technology to increase the accessibility of eye care. ${ }^{42}$ The results of this review cannot be directly disseminated to participants of the included primary studies. However, we will try to communicate the results via peer-reviewed journals and scientific conferences.

\section{DISCUSSION}

With its growing incidence and burden on healthcare systems globally, diabetes has emerged as a leading cause of vision loss through DR and DME. ${ }^{1}$ Yet, in most cases, severe vision loss from diabetes is preventable. Patients with diabetes, irrespective of the type of diabetes, require regular retinal screening for early detection and timely treatment of $\mathrm{DR} .{ }^{43}$ Screening for retinopathy is conventionally done in a doctor's office by trained healthcare professionals. Smartphone-based retinal imaging has lately emerged as a potentially more effective, affordable and accessible alternative for identification of DR. A Pew Research Center report for 2017-2018 indicates a smartphone penetration rate of $42 \%$ in emerging and developing economies, and $72 \%$ for advanced economies. ${ }^{44}$ Coupled with AI grading or telemedicine networks, smartphone ophthalmoscopy is possibly ideal for DR mass screening in resource-constrained countries. Furthermore, non-specialised healthcare professionals may be able to operate this technology, enhancing its feasibility and convenience. However, smartphone ophthalmoscopy has its limitations. The image quality may be limited by the stability of examiners' hands, small pupil size or unwanted corneal reflections. ${ }^{45}$ Nevertheless, in one study, smartphone ophthalmoscopy captured equal or better quality photographs compared with traditional fundus cameras. ${ }^{46}$ In light of the above facts, there is a need for a thorough evaluation of the diagnostic test accuracy of the use of smartphone ophthalmoscopy for DR detection.

In this review, our aim is to collate all evidence on the diagnostic test accuracy of smartphone ophthalmoscopy for DR detection. As this is a new and fast advancing research field, we will employ a broad inclusion criteria. We will use a scoping review methodology to this end and thoroughly perform literature searches, screening, data extraction, study quality assessments and data synthesis. Our aim is to provide a robust evidence base that will allow for reliable recommendations for policy and future research on the use of smartphones for DR detection. 
Contributors LTC and HS conceived the idea for the project. CHT, W-HQ and LTC planned, conceived and designed the protocol. $\mathrm{CHT}$ designed and piloted the search strategy. HS and CT critically reviewed and provided feedback.

Funding This research is supported by the Lee Kong Chian School of Medicine, Nanyang Technological University Singapore Start-Up Grant.

Competing interests None declared.

Patient consent for publication Not required.

Provenance and peer review Not commissioned; externally peer reviewed.

Open access This is an open access article distributed in accordance with the Creative Commons Attribution Non Commercial (CC BY-NC 4.0) license, which permits others to distribute, remix, adapt, build upon this work non-commercially, and license their derivative works on different terms, provided the original work is properly cited, appropriate credit is given, any changes made indicated, and the use is non-commercial. See: http://creativecommons.org/licenses/by-nc/4.0/.

ORCID iD

Choon Han Tan http://orcid.org/0000-0002-5448-3365

\section{REFERENCES}

1 Cheung N, Mitchell P, Wong TY. Diabetic retinopathy. The Lancet 2010;376:124-36

2 Lee R, Wong TY, Sabanayagam C. Epidemiology of diabetic retinopathy, diabetic macular edema and related vision loss. Eye and Vision 2015;2.

3 Rodrigues GB, Abe RY, Zangalli C, et al. Neovascular glaucoma: a review. Int J Retin Vitr 2016;2.

4 Cohen SR, Gardner TW. Diabetic retinopathy and diabetic macular edema. Dev Ophthalmol 2016;55:137-46.

5 Stewart MW. Treatment of diabetic retinopathy: recent advances and unresolved challenges. World J Diabetes 2016;7:333-41.

6 Dowler JGF. Laser management of diabetic retinopathy. J R Soc Med 2003:96:277-9.

7 Gupta N, Mansoor S, Sharma A, et al. Diabetic retinopathy and VEGF. Open Ophthalmol J 2013;7:4-10.

8 Photocoagulation for diabetic macular edema. early treatment diabetic retinopathy study report number 1 . early treatment diabetic retinopathy study Research Group. Arch Ophthalmol 1985;103:1796-806.

9 Early vitrectomy for severe vitreous hemorrhage in diabetic retinopathy. four-year results of a randomized trial: diabetic retinopathy vitrectomy study report 5 . Arch Ophthalmol 1990;108:958-64.

10 Mohamed Q, Gillies MC, Wong TY. Management of diabetic retinopathy: a systematic review. JAMA 2007;298:902-16.

11 Ellis D, Burgess PI, Kayange P. Management of diabetic retinopathy. Malawi Med J 2013;25:116-20.

12 Ghasemi Falavarjani K, Wang K, Khadamy J, et al. Ultra-wide-field imaging in diabetic retinopathy; an overview. J Curr Ophthalmol 2016;28:57-60.

13 Panwar N, Huang P, Lee J, et al. Fundus Photography in the 21st Century--A Review of Recent Technological Advances and Their Implications for Worldwide Healthcare. Telemed J E Health 2016;22:198-208.

14 Shields CL, Materin M, Shields JA. Panoramic imaging of the ocular fundus. Arch Ophthal 2003;121:1603-7.

15 Lira RPC, Oliveira CLdeA, Marques MVRB, et al. Adverse reactions of fluorescein angiography: a prospective study. Arq Bras Oftalmol 2007;70:615-8.

16 Ahmed J, Ward TP, Bursell S-E, et al. The sensitivity and specificity of nonmydriatic digital stereoscopic retinal imaging in detecting diabetic retinopathy. Diabetes Care 2006;29:2205-9.

17 Goh JKH, Cheung CY, Sim SS, et al. Retinal imaging techniques for diabetic retinopathy screening. J Diabetes Sci Technol 2016;10:282-94.

18 Massin P, Girach A, Erginay A, et al. Optical coherence tomography: a key to the future management of patients with diabetic macular oedema. Acta Ophthalmol Scand 2006;84:466-74.

19 Hong SC. 3D printable retinal imaging adapter for smartphones could go global. Graefes Arch Clin Exp Ophthalmol 2015;253:1831-3.

20 Jones S, Edwards RT. Diabetic retinopathy screening: a systematic review of the economic evidence. Diabet Med 2010;27:249-56.

21 Owolabi B, Odugbemi T, Odeyemi K, et al. mHealth: knowledge and use among doctors and nurses in public secondary health- care facilities of Lagos, Nigeria. Journal of Clinical Sciences 2018;15:27-31.

22 Jiang $\mathrm{F}$, Jiang $\mathrm{Y}$, Zhi H, et al. Artificial intelligence in healthcare: past, present and future. Stroke Vasc Neurol 2017;2:230-43.

23 Gulshan V, Peng L, Coram M, et al. Development and validation of a deep learning algorithm for detection of diabetic retinopathy in retinal fundus Photographs. JAMA 2016;316:2402-10.

24 Walton OB, Garoon RB, Weng CY, et al. Evaluation of automated Teleretinal screening program for diabetic retinopathy. JAMA Ophthalmol 2016;134:204-9.

25 Rajalakshmi R, Subashini R, Anjana RM, et al. Automated diabetic retinopathy detection in smartphone-based fundus photography using artificial intelligence. Eye 2018;32:1138-44.

26 Shi L, Wu H, Dong J, et al. Telemedicine for detecting diabetic retinopathy: a systematic review and meta-analysis. $\mathrm{Br} J$ Ophthalmol 2015;99:823-31.

27 Horton MB, Silva PS, Cavallerano JD, et al. Clinical components of telemedicine programs for diabetic retinopathy. Curr Diab Rep 2016;16:129.

28 Bolster NM, Giardini ME, Bastawrous A. The diabetic retinopathy screening workflow: potential for smartphone imaging. J Diabetes Sci Technol 2015;10:318-24.

29 Micheletti JM, Hendrick AM, Khan FN, et al. Current and next generation portable screening devices for diabetic retinopathy. $J$ Diabetes Sci Technol 2016;10:295-300.

30 Fenner BJ, Wong RLM, Lam W-C, et al. Advances in retinal imaging and applications in diabetic retinopathy screening: a review. Ophthalmol Ther 2018;7:333-46.

31 Vilela MAP, Valença FM, Barreto PKM, et al. Agreement between retinal images obtained via smartphones and images obtained with retinal cameras or fundoscopic exams - systematic review and metaanalysis. Clin Ophthalmol 2018;12:2581-9.

32 Levac D, Colquhoun H, O'Brien KK. Scoping studies: advancing the methodology. Implementation Sci 2010;5.

33 Arksey H, O'Malley L. Scoping studies: towards a methodological framework. Int J Soc Res Methodol 2005;8:19-32.

34 Tricco AC, Lillie E, Zarin W, et al. PRISMA extension for scoping reviews (PRISMA-ScR): checklist and explanation. Ann Intern Med 2018;169:467-73.

35 Higgins JPT, Green S, eds. Cochrane Handbook for Systematic Reviews of Interventions Version 5.1.0. The Cochrane Collaboration, 2011. www.handbook.cochrane.org

36 Shanmugam M, Mishra D, Madhukumar R, et al. Fundus imaging with a mobile phone: a review of techniques. Indian $\mathrm{J}$ Ophthalmol 2014;62:960-2.

37 The Nordic Cochrane Centre. Review Manager (RevMan) [Computer program]. Version 5.3. Copenhagen: The Cochrane Collaboration, 2014

38 Whiting PF, Rutjes AWS, Westwood ME, et al. QUADAS-2: a revised tool for the quality assessment of diagnostic accuracy studies. Ann Intern Med 2011;155:529-36.

39 Pham MT, Rajić A, Greig JD, et al. A scoping review of scoping reviews: advancing the approach and enhancing the consistency. Res. Syn. Meth. 2014;5:371-85.

40 Daudt HML, van Mossel C, Scott SJ. Enhancing the scoping study methodology: a large, inter-professional team's experience with Arksey and O'Malley's framework. BMC Med Res Methodol 2013;13:48

41 Deeks JJ, Macaskill P, Irwig L. The performance of tests of publication bias and other sample size effects in systematic reviews of diagnostic test accuracy was assessed. J Clin Epidemiol 2005;58:882-93.

42 Garms RC. The global initiative: Launch of vision 2020. Community Eye Health 1998;11:56-7.

43 Vashist P, Gupta N, Singh S, et al. Role of early screening for diabetic retinopathy in patients with diabetes mellitus: an overview. Indian $J$ Community Med 2011;36:247-52.

44 Poushter J, Bishop C, Chwe H. Social media use continues to rise in developing countries but plateaus across developed ones: Pew research center, 2018. Available: http://assets.pewresearch.org/wpcontent/uploads/sites/2/2018/06/15135408/Pew-Research-Center Global-Tech-Social-Media-Use_2018.06.19.pdf [Accessed Nov 2018].

45 Nazari Khanamiri H, Nakatsuka A, El-Annan J. Smartphone fundus photography. JoVE 2017;125.

46 Adam MK, Brady CJ, Flowers AM, et al. Quality and diagnostic utility of mydriatic smartphone photography: the smartphone ophthalmoscopy reliability trial. Ophthalmic Surg Lasers Imaging Retina 2015;46:631-7. 